\title{
Random Growth Models
}

Note from Editor: The organizers of the 2017 Joint Mathematics Meetings Short Course on Random Growth Models have kindly agreed to provide this introduction to the subject. See p. 1087 for more information on the JMM 2017 Short Course.

\section{Michael Damron, Firas Rassoul-Agha, and Timo Seppäläinen}

$\mathrm{I}$ rregular and stochastic growth is all around us: tumors, bacterial colonies, infections, fluid spreading in a porous medium, propagating flame fronts. The study of simplified mathematical models of stochastic growth began in probability theory half a century ago. Quite serendipitously these models have turned out to be extremely hard to analyze. They have inspired innovative probability theory and have led to new connections between probability and other parts of mathematics.

This brief overview discusses two classes of such mathematical models, namely undirected first-passage percolation (FPP) and directed last-passage percolation (LPP) on the $d$-dimensional integer lattice $\mathbb{Z}^{d}$. The basic idea is the following. An infection starts at the origin and progresses along nearest-neighbor lattice paths. Depending on the model, admissible paths are either directed, so that each step is forced to be one of the standard basis vectors $e_{i}$, or undirected. The time it takes for the infection to reach a given lattice point is determined by random passage times assigned either to edges or to vertices of the lattice. FPP seeks the path of minimal passage time, while LPP maximizes passage time.

In (undirected) first-passage percolation, we give each nearest-neighbor edge $e$ of the lattice $\mathbb{Z}^{d}$ a nonnegative random passage time $t_{e}$. Collectively the random variables $\left\{t_{e}\right\}$ are typically independent and identically distributed. The model is parametrized by the common probability distribution $\mu$ of the $t_{e} s$. The passage time of a lattice path $\gamma$ (a sequence of consecutive edges) is $T(\gamma)=\sum_{e \in \gamma} t_{e}$. The passage time between points $x$ and $y$ is $T(x, y)=$

Michael Damron is assistant professor in the School of Mathematics at Georgia Tech. His email address is mdamron6@gatech. edu.

Firas Rassoul-Agha is professor of mathematics at the University of Utah. His email address is fi ras@math. utah. edu.

Timo Seppäläinen is the John and Abigail Van Vleck Chair of Mathematics at the University of Wisconsin-Madison. His email address is seppa1ai@math. wisc . edu.

For permission to reprint this article, please contact: reprint-permission@ams.org.

DOI: http://dx.doi.org/10.1090/noti1400

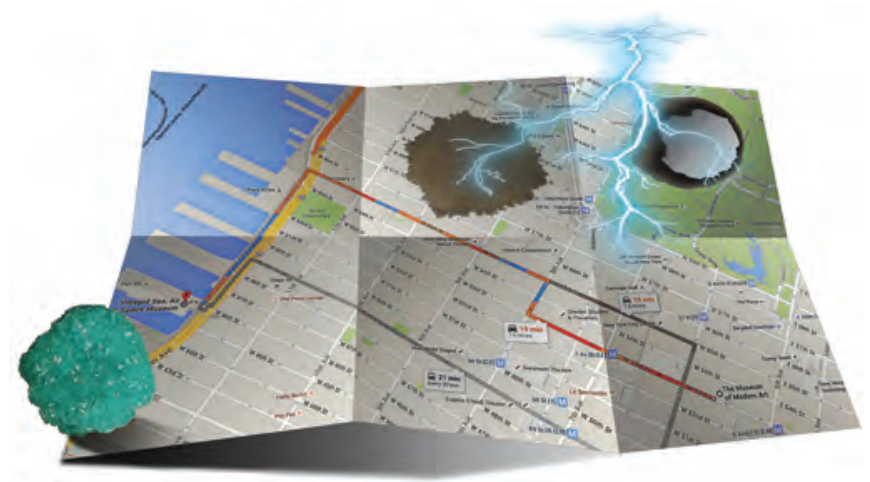

Figure 1. A traffic map ${ }^{1}$ showing an optimal (geodesic) path between two locations and color-coding the edge weights along the path. Lightning ${ }^{2}$ explores every path available to it in space and strikes along the path of least electrical resistance, much like the optimal paths discussed in the section "Geodesics". Shapes such as the ones in the section "Limit Shapes" and Figure 3 are ubiquitous in nature, e.g. stains, burning regions, and growing crystals. ${ }^{3}$

$\inf _{y: x \rightarrow y} T(\gamma)$, the minimal passage time of a path between $x$ and $y$. If none of the $t_{e}$ s are zero, then $T$ is a random metric on $\mathbb{Z}^{d}$. The infection starts at the origin, and the set of infected sites at time $t \geq 0$ is

$$
B(t)=\left\{x \in \mathbb{Z}^{d}: T(0, x) \leq t\right\} .
$$

The leftmost picture in Figure 2 illustrates the optimal paths from the origin to the lattice points in $B(t)$. The middle picture in Figure 3 shows a larger FPP cluster, with an experimental cluster on the right for comparison. A thorough recent survey of the mathematics of FPP is provided by Auffinger et al. [1].

\footnotetext{
${ }^{1}$ Generated using Google Maps.

${ }^{2}$ Courtesy of James McGhee.

${ }^{3}$ Courtesy of dans-1e-townhouse . blogspot . com .
} 

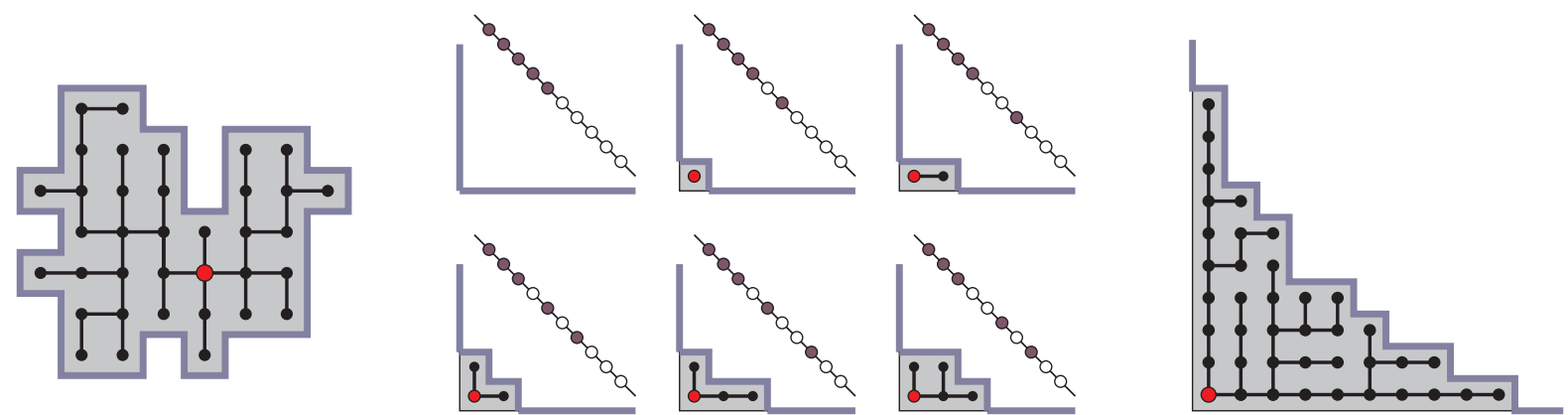

Figure 2. The leftmost picture is a snapshot of the growing FPP cluster on the plane at some fixed time $t>0$. Bullets mark lattice points $x$ with $T(0, x) \leq t$. The origin is distinguished with the red bullet. The gray region is the fattened set $B(t)+[-1 / 2,1 / 2]^{2}$. The bold black edges are the paths of minimal passage time from the origin. They are not forced to be directed. The middle and rightmost pictures depict a possible early evolution of the corner growth model on the first quadrant of the plane. The origin is again the red bullet. Optimal paths are all directed. The antidiagonals illustrate the mapping of the corner growth model to an interacting particle system of central importance, namely the totally asymmetric simple exclusion process (TASEP) [2]. Whenever a point is added to the growing cluster, a particle (solid purple circle) switches places with the hole (open circle) to its right.

Another much-studied model is the corner growth model, which is directed last-passage percolation on the planar square lattice. Passage times are conventionally assigned to vertices. Admissible paths take only $e_{1}$ and $e_{2}$ steps, and $T(0, x)$ is the maximal passage time of a path from 0 to $x$. This model stands at the nexus of several disciplines: queueing theory, interacting particle systems, integrable systems, and representation theory. Its evolution is illustrated in the middle and right pictures of Figure 2 and in the leftmost picture of Figure 3.

We address below three fundamental questions about the growth of $B(t)$ :

(1) Does $B(t)$ acquire a definite shape?

(2) How does $B(t)$ fluctuate randomly around its long-term shape?

(3) How can we describe the geometry of optimal paths (geodesics) for $T$ ?

\section{Limit Shapes}

The basic law of large numbers of the subject is the shape theorem. For first-passage percolation (FPP), it was initially proved by Richardson (1973) and then refined considerably by Cox-Durrett (1981) and Kesten (1986). To give the random infected set $B(t)$ positive volume in $\mathbb{R}^{d}$, replace it with the fattened sum set $B(t)+[-1 / 2,1 / 2]^{d}$. Then there is a nonrandom, convex, compact set $B_{\mu}$ in $\mathbb{R}^{d}$ such that $t^{-1} B(t)$ converges almost surely to $B_{\mu}$ in Hausdorff distance, as $t \rightarrow \infty$. $B_{\mu}$ depends on the common probability distribution $\mu$ of the weights $t_{e}$. It is symmetric about the axes and has nonempty interior. In Figure 4 we can discern that the growing clusters approach a limiting shape.

What does $B_{\mu}$ look like in FPP? For a trivial point of comparison, note that with constant edge weights the limit shape is the $\ell^{1}$ unit ball, which is a diamond with sharp corners and flat faces and edges. The randomness of the weights is expected to smooth out and curve the limit shape. If $\mu$ is continuous, it is predicted that $B_{\mu}$ is strictly convex with a uniformly positively curved, differentiable boundary. There is little progress toward verifying these properties. It is not even known that $B_{\mu}$ is not a polygon (that is, has infinitely many extreme points), except in the case of some atomic $\mu$ in two dimensions. Contrary to good taste, it is believed that $B_{\mu}$ is not a Euclidean ball for typical $\mu$. This has been proved in high dimensions. There are no results that say that generally the shape is not a ball in low dimensions, but this has been verified for certain distributions.

For the first fifty years of the subject, no general descriptions of limit shapes existed beyond their limit definitions. Recently Krishnan and independently Georgiou, Rassoul-Agha, and Seppäläinen discovered variational formulas for the limiting time constant $g(x)=$ $\lim _{n \rightarrow \infty} n^{-1} T(0,\lfloor n x\rfloor)$. These variational formulas live on complicated infinite-dimensional spaces of functions or measures, and consequently extracting information from them is a difficult problem in itself.

Among directed models in two dimensions there are special exactly solvable ones where fortuitous coincidences of combinatorics and probability permit a closed-form evaluation of the limit shape. The oldest such is the corner growth model with exponentially distributed weights (the leftmost picture of Figure 3).

\section{Shape Fluctuations}

A law of large numbers raises the question of fluctuations, that is, the difference between $t^{-1} B(t)$ and $B_{\mu}$. In Figures 3 and 4 we see the roughness of the growing boundary that results from the stochastic fluctuations in the growth.

One can split this error into a random part and a nonrandom part. The random part is represented by the discrepancy between $T(0, x)$ and its mean $\mathbb{E} T(0, x)$ when $x$ is large. The nonrandom part is the discrepancy between $\mathbb{E} T(0, x)$ and the limiting value $g(x)$. It is predicted that 

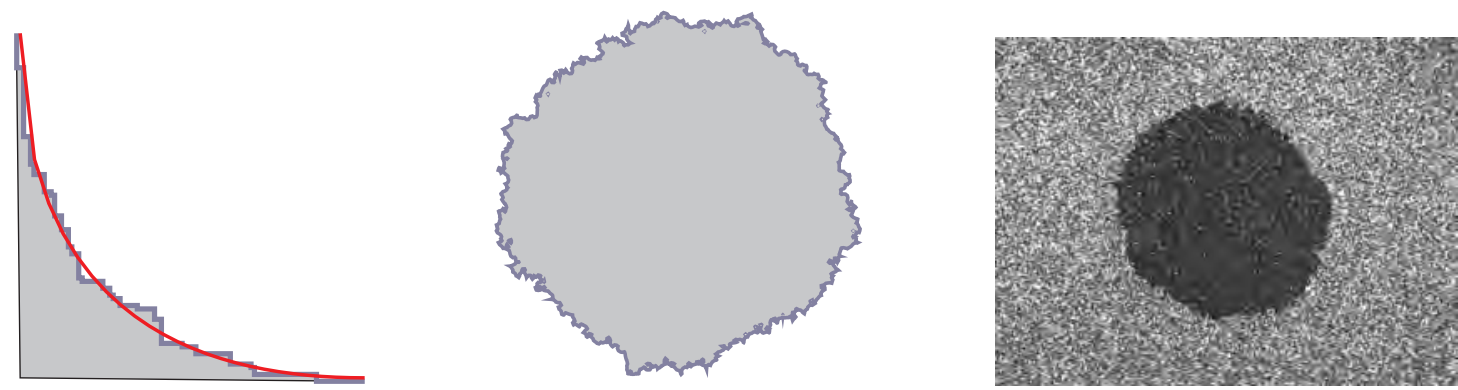

Figure 3. Left: The corner growth model with exponentially distributed vertex weights with mean 1 . The gray region is a simulation of the scaled growing set $t^{-1}\left(B(t)+[-1 / 2,1 / 2]^{2}\right)$ at time $t=160$. Its boundary (the thick blue line) approximates the red limit curve $\sqrt{x}+\sqrt{y}=1$, as first proved by Rost in 1981. Middle: A simulation of the scaled set $t^{-1}\left(B(t)+[-1 / 2,1 / 2]^{2}\right)$ at time $t=40$ for undirected first-passage percolation with edge weights that are exponential with mean 1 . Such shapes are ubiquitous in nature, e.g. stains, fire fronts, and surfaces of growing crystals. Right: A growing interface in liquid-crystal turbulence. Takeuchi and Sano demonstrated that these experimental interfaces possess the same KPZ statistics as the corner growth model with exponential weights. It is believed that this behavior is universal among two-dimensional FPP and LPP models.

Directed LPP with Exp(1) weights

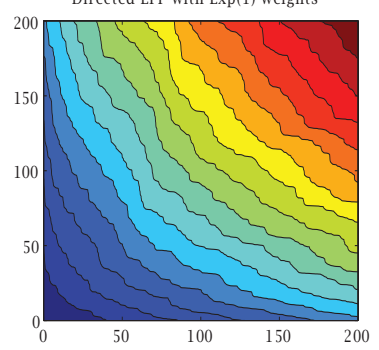

Standard FPP with Exp(1) weights

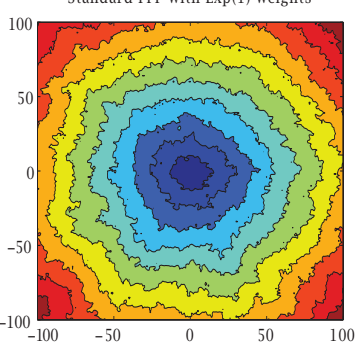

Directed LPP with Exp(1) weights

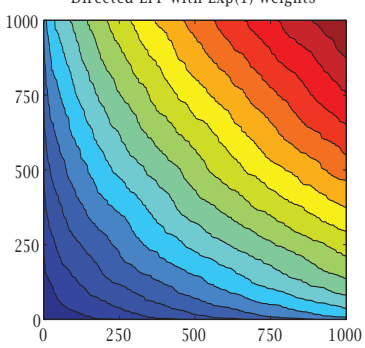

Standard FPP with Exp(1) weights

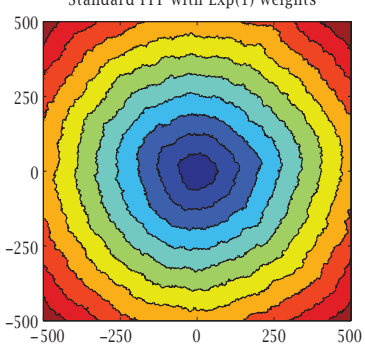

Directed LPP with Ber (0.75) weights

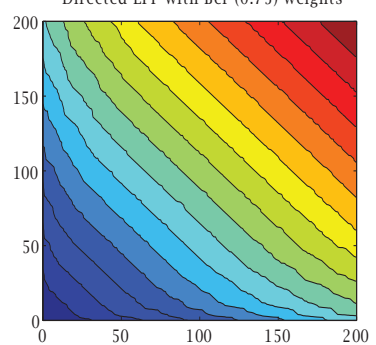

Standard FPP with $1+$ B er(0.35) weights

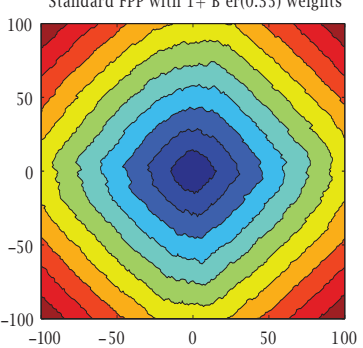

Directed LPP with Ber (0.75) weights

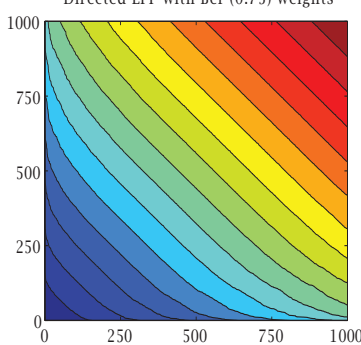

Standard FPP with $1+$ Ber $(0.35)$ weights

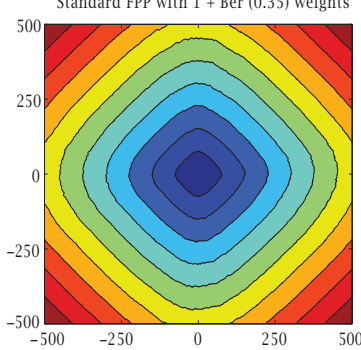

Figure 4. Growth of the infected region with exponential and Bernoulli distributed weights. Top row directed LPP, bottom row undirected FPP, colors mark successive level sets of $T(0, x)$, and lattice distances are marked on the axes. The right-hand pictures illustrate the Durrett-Liggett flat edge. A flat edge appears in the limit shape when the optimal weight values are frequent enough to form an infinite directed connected component on $\mathbb{Z}^{d}$. This phenomenon is called oriented percolation.

there is a dimension-dependent exponent $\zeta$ such that the typical deviation of $T(0, x)$ from $\mathbb{E} T(0, x)$ is of order $|x|^{\zeta}$. One way to phrase this is to assert that $\operatorname{Var}[T(0, x)]$ is of order $|x|^{2 \zeta}$.

What is predicted or proved about this exponent $\zeta$ ? Physicists believe that at least for low dimensions, $\zeta$ is positive and strictly less than $1 / 2$. This latter behavior is called "subdiffusivity" and is associated with other predicted aspects of the model, like abundance of near-optimizing paths. The best rigorous bounds to date were established by Kesten (1993), stating that $0 \leq \zeta \leq 1 / 2$ for all dimensions. In two dimensions there are logarithmic lower bound corrections $\operatorname{Var}[T(0, x)] \geq c \log |x|$ by Pemantle-Peres (1993) and Newman-Piza (1995), and for $d \geq 2$ there are similar corrections to the upper bound: $\operatorname{Var}[T(0, x)] \leq c|x| / \log |x|$ by Benjamini-Kalai-Schramm (2003), Benaïm-Rossignol (2008), and Damron-Hanson-Sosoe (2014).

For a handful of exactly solvable two-dimensional directed models, there are precise results that give $\zeta=$ $1 / 3$ and even the limiting distribution for the scaled discrepancy $n^{-1 / 3}(T(0,\lfloor n x\rfloor)-n g(x))$ as $n \rightarrow \infty$. These phenomena are studied by mathematicians and physicists under the rubric Kardar-Parisi-Zhang universality [2]. It is 

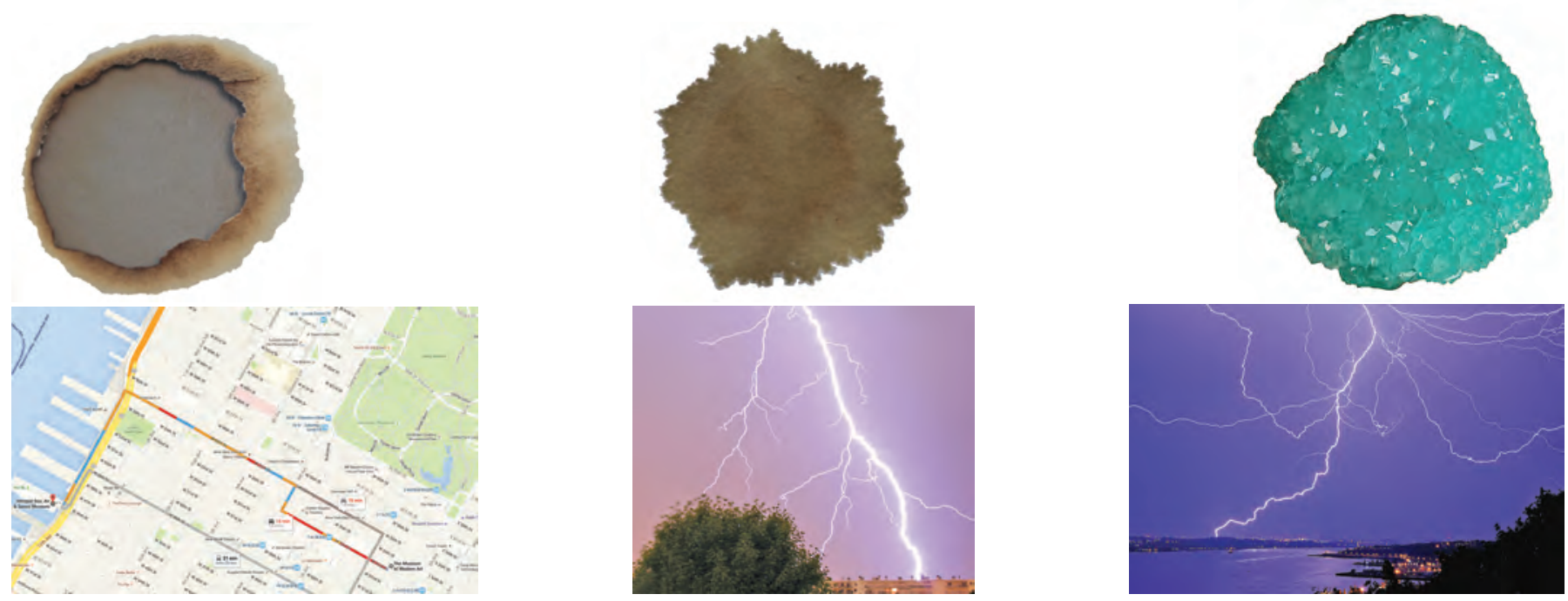

Figure 5. Top row, left to right: A burnt-out hole in a paper, a coffee stain that percolated on a piece of paper, and a Borax crystal. ${ }^{4}$ A traffic map ${ }^{5}$ showing an optimal (geodesic) path between two locations and color-coding the edge weights along the path. Lightning ${ }^{6,7}$ explores every path available to it in space and strikes along the path of least electrical resistance, much like the optimal paths discussed in the section "Geodesics".

expected that, subject to a mild moment assumption on the random passage times, all two-dimensional FPP and LPP models obey KPZ universality.

\section{Geodesics}

When $\mu$ is continuous, optimal paths or geodesics between points are almost surely unique. In 1995 Newman introduced an approach to infinite geodesics (infinite paths whose segments are geodesics) that starts with the infection tree $\mathcal{T}$ defined as the union of the geodesics from the origin to all the lattice points. Somewhat trivially, the analogous tree in $\mathbb{R}^{d}$ under the Euclidean metric would have an infinite ray from 0 in each direction and hence uncountably many disjoint infinite paths from 0 . A similar property is predicted for these growth models, at least in low dimension. One expects an infinite path in $\mathcal{T}$ from 0 in each fixed direction, and so uncountably many such distinct paths. (Two paths are distinct if they separate eventually.)

In undirected FPP, these statements are far from being proved. Hoffman showed in 2008 that in general $\mathcal{T}$ has at least four infinite eventually disjoint paths starting from 0 . But not even the existence of a single continuous distribution $\mu$ such that a path in $\mathcal{T}$ has an asymptotic direction is known. As one might expect, in directed planar models geodesics are better understood. In exactly solvable cases, such as the exponential corner growth model of Figure 3, the aforementioned geodesic conjecture can be proved in full.

Suppose now we start infections from two points on the lattice and let these infections compete for space. The

\footnotetext{
${ }^{4}$ Courtesy of dans-1e-townhouse.blogspot.com.

${ }^{5}$ Generated using Google Maps.

${ }^{6}$ By Axel Rouvin via Wikimedia Commons.

${ }^{7}$ By Jp Marquis via Wikimedia Commons.
}
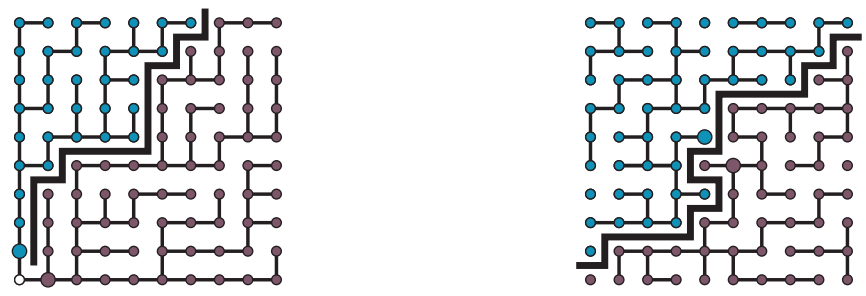

Figure 6. Left: The full tree of infection in the corner growth model. The origin is the open circle at the bottom left. The solid black line marks the competition interface that separates the two competing infections that grow from points $(1,0)$ and $(0,1)$ marked with larger circles. Globally, the competition interface points in a precise but randomly chosen direction whose probability distribution can be derived in exactly solvable cases. Right: Competition in undirected FPP. The competition interface separates the infection trees originating from $(1,0)$ and $(0,1)$ marked with larger circles.

growth model has become a model of competition. Figure 6 shows the competition interface between two infections emanating from $(1,0)$ and $(0,1)$, both in the directed corner growth model, and for undirected first-passage percolation.

For competition in undirected first-passage percolation, there are two possible scenarios: either both infections have a chance of growing indefinitely (coexistence) or ultimately one encircles the other for sure. Conditions for these two occurrences are a subject of ongoing research. In coexistence, the competition interface is a doubly infinite path with two random asymptotic directions. 


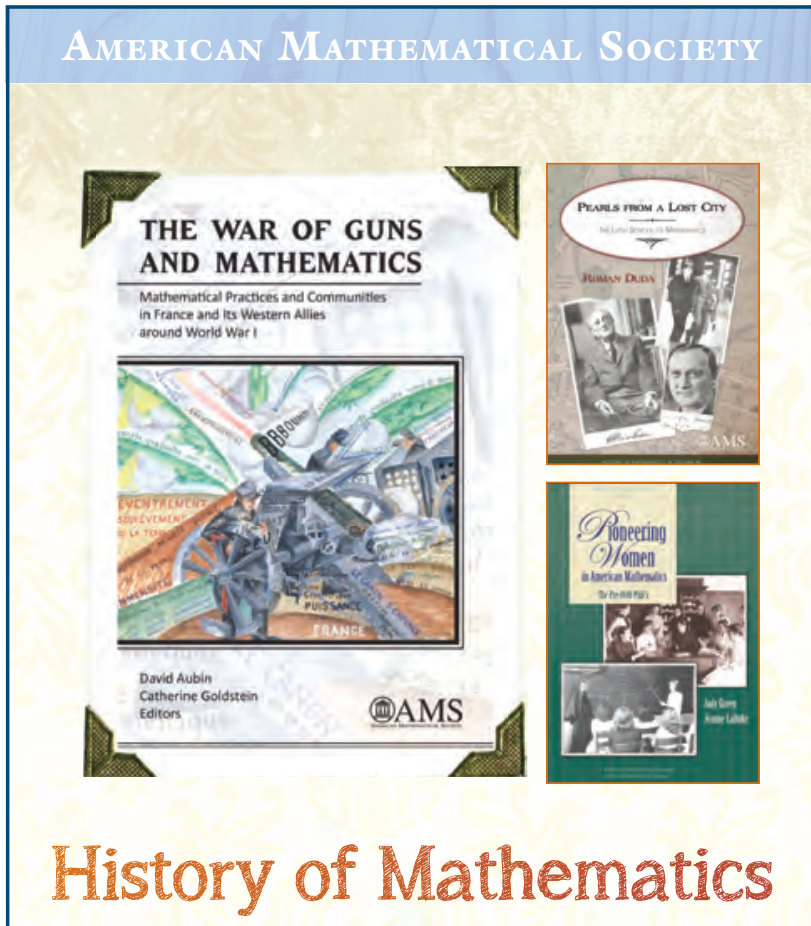

The titles in this series offer compelling historical perspectives on the individuals and communities that have profoundly influenced mathematics development. Each book constitutes a valuable addition to a historical or mathematical book collection.
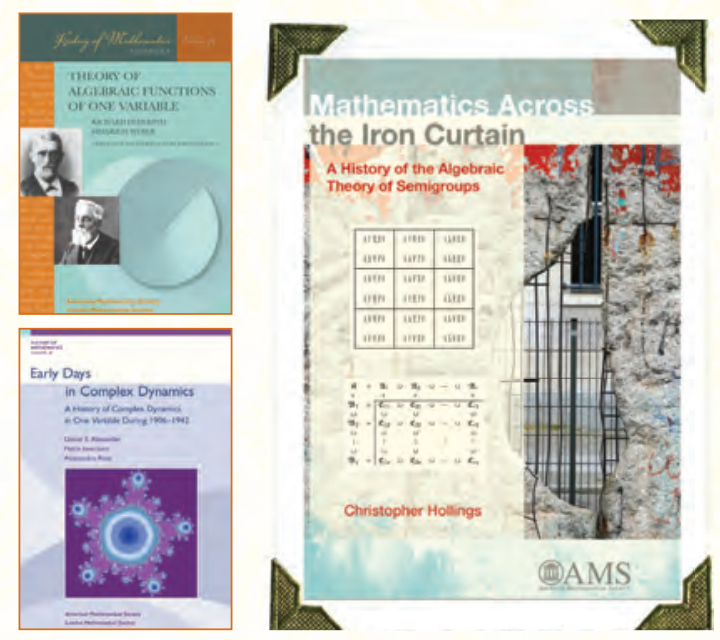

bookstore.ams.org/HMATH

(i.i)AMS Bookstore, (800) $321-4267$ (U.S. \& Canada) (401)455-4000 (Worldwide)

bookstore.ams.org
The behavior of the competition interface can be quantified with Busemann functions. This notion from metric geometry was introduced into percolation by Newman and Hoffman. In our context, Busemann functions are equal to the limits $b(x, y)=\lim _{v \rightarrow \infty}(T(x, v)-T(y, v))$ as a point $v$ recedes to infinity in a particular direction. In exactly solvable models, this limit can be proved to exist, and its behavior is understood completely. Busemann functions can be used to construct stationary growth models whose global probability laws are invariant under spatial translations and whose growth is linear on average. Such versions of stochastic processes are very useful for proofs and explicit calculations. A recent development in the field, in the work of Damron-Hanson and GeorgiouRassoul-Agha-Seppäläinen, is that Busemann functions have provided a route toward verifying versions of the geodesic conjectures stated above.

\section{References}

[1] Antonio Auffinger, Michael Damron, and Jack Hanson, 50 years of first passage percolation, 2015. arXiv:1511.03262

[2] Ivan Corwin, Kardar-Parisi-Zhang universality, Notices Amer. Math. Soc. 63 (2016), no. 3, 230-239. MR 3445162

THE AUTHORS

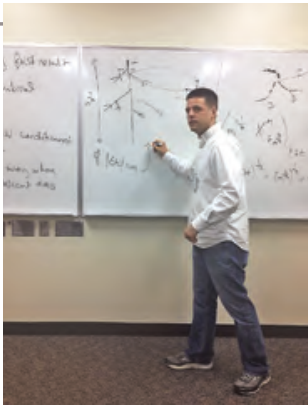

Michael Damron

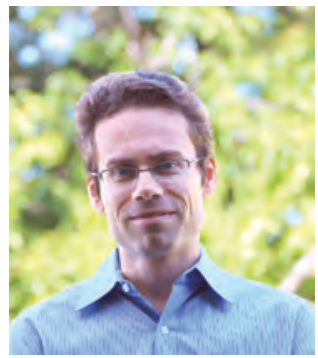

Firas Rassoul-Agha

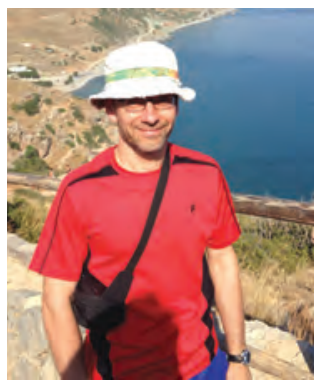

Timo Seppäläinen 